\title{
Advanced Approaches to Functional Glasses
}

\author{
Satoru INOUE \\ Advanced Materials Laboratory, National Institute for Materials Science, 1-1, Namiki, Tsukuba-shi, Ibaraki 305-0044
}

\section{ガラスの機能化手法}

井上 悟

物質·材料研究機構物質研究所, 305-0044 茨城県つくば市並木 1-1

\begin{abstract}
Two key technologies were reviewed to propose effective approaches to functional glasses. One is combinatorial glass synthesis system. The combinatorial glass synthesis system consists of automatic batch preparation apparatus and two types of automatic apparatuses for melting of glass batches in crucibles. The combinatorial system was effective to search new glass forming systems and to speed up preparation of glass samples. The system was useful for the developments of bulk functional glasses based on doping of ions or compounds. The other is a new technique based on anodization of aluminum. The anodic oxidation of sputtered aluminum film on glass surfaces was applied to preparation of alumina nanostructures possessing nanopore arrays on glass surfaces. Oxides or metallic compounds could be introduced into nanopores using sol-gel technique or electro deposition technique. The introduction of $\mathrm{TiO}_{2}$ by sol-gel process yielded high performance photo catalysis showing decomposition rate of acetaldehyde higher by about 13 times than that of P-25 commercial high performance $\mathrm{TiO}_{2}$ powder catalysis. The impregnation of $\mathrm{Ni}$ metal into nanopores by electro deposition gave high density magnetic rod array of about $600 \mathrm{G} /$ inch $^{2}$, suggesting that the process would be applicable to the development of high density magnetic recording media. The new process based on anodic oxidation of sputtered aluminum was very effective to develop functional nanostructure layers on glass surfaces.

[Received May 27, 2003]
\end{abstract}

Key-words : Functional glass, Combinatorial methodologies, Glass formation, Melt-quenching method, Anodic oxidation, Sputtered aluminum, Sol-gel technique, Electro deposition

1. Introduction

$\mathbf{I}^{\mathrm{N}}$ the developments of functional glasses, glass researchers have two choices of approaches; (a) one is the doping or addition of active elements or compounds into glass matrices, and (b) the other is preparation of functional thin layer on glass substrates. Case (a) corresponds to the preparation of ordinary bulk glasses containing ions or compounds which give high/low refraction of light, high/low dispersion of refractive index, high non-linear refractive index, fluorescence emission, photochromism, ${ }^{1)}$ thermochromism, ${ }^{2)}$ photoconduction, ion conduction, color filters, electron conduction etc. Case (b) produces glasses like multicoated mirrors/filters, LCD display glasses, HDD disks etc. The phenomena such as crystallization, phase separation, ion exchange and glass transition, which are the unique properties of glasses, are used to modify the structures of glasses in both of the approaches.

The case (a) normally forces the researchers to do many experiments including search of glass forming systems and/ or optimization of the compositions of glasses so as to generate sharp functions. In the case (b), glasses do not contribute to generation of the functions and play the roles of supporting the functional films. For approaching functional glasses, the speed up of the experiments to search new glass forming systems would be one of the key technologies in the case (a) and a simple and massproduction type preparation method to build up nanostructures on glass surfaces would be one of the key technologies in the case (b).

In this report, two key technologies to promote developments of functional glasses are reviewed. One is the combinatorial glass synthesis methodologies and the other is a new preparation method of nanostructures on glass surfaces through anodic oxidation of sputtered aluminum thin films.

\section{Combinatorial glass synthesis system}

2.1 Combinatorial glass synthesis methodologies

The construction of the system is shown in Fig. 1 as an illustration. The system consists of laboratory common glass synthesis processes including batch preparation, melting of batches and casting/cooling of melts. The glass batches can be prepared with the Combinatorial Glass Batch Preparation Apparatus ${ }^{3)}$ at a rate of 12-24 batches for one time in a 4-component system. Figure 2 shows the possible preparation methods taken into consideration. Method A is derived from the composition spread technique that is typical combinatorial technique among the researchers in the field of semiconductors. ${ }^{4)}$ In this method, laser beam

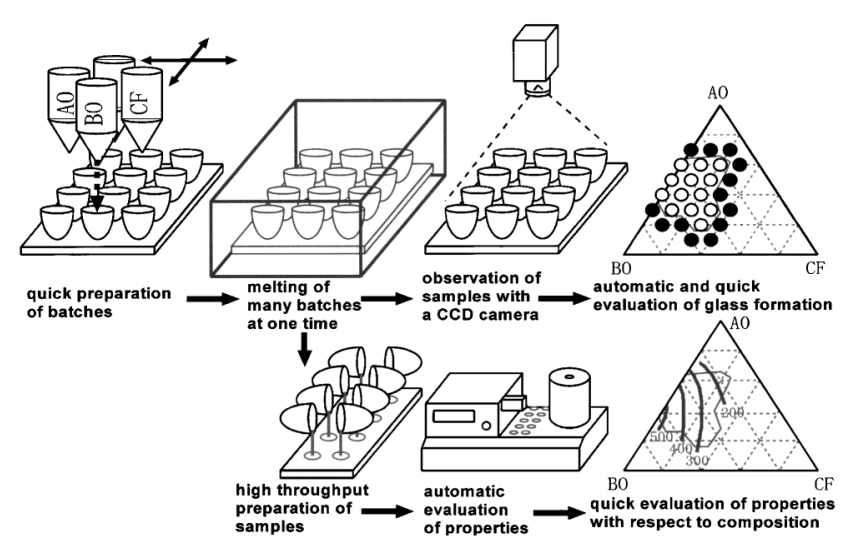

Fig. 1. Illustration of combinatorial glass synthesis system. 


\begin{tabular}{|c|c|c|c|}
\hline & Method & Weight & Features \\
\hline $\mathbf{A}$ & $\begin{array}{l}\text { melting of } \\
\text { condensed films } \\
\text { on a substrate } \\
\text { laser beam }\end{array}$ & $\sim 10 \mathrm{mg}$ & $\begin{array}{l}\text { *very small scale of batch } \\
\text { *hard to collect samples } \\
\text { *suitable for high throughput } \\
\text { screening like composition } \\
\text { spread technique } \\
\text { *laser beam heating is } \\
\text { recommended }\end{array}$ \\
\hline B & $\begin{array}{l}\text { melting in small } \\
\text { containers } \\
\text { batch }\end{array}$ & $\sim 1 \mathrm{~g}$ & $\begin{array}{l}\text { *easy to set up parallel } \\
\text { arrangements } \\
\text { *possible to employ noble metals } \\
\text { or carbon as containers } \\
\text { *possible to recover the samples } \\
\text { by employing the containers } \\
\text { which are not wetted with melts }\end{array}$ \\
\hline C & $\begin{array}{l}\text { melting in } \\
\text { crucibles }\end{array}$ & $\underset{10 \mathrm{~g}}{1 \mathrm{~g} \sim}$ & $\begin{array}{l}\text { *easy to collect samples } \\
\text { *a little hard to set up } \\
\text { parallel arrangements } \\
\text { *easy to check the glass } \\
\text { formation } \\
\text { "possible to employ noble metals } \\
\text { or carbon as containers }\end{array}$ \\
\hline
\end{tabular}

Fig. 2. Possible ways for combinatorial glass synthesis.

heating is necessary to keep the heating and melting area as small as possible, in other words, to keep the melt droplet as small as possible. This method enables us to execute high throughput screening of the composition that can be converted into glasses, but it is hard to collect the samples. Method B is suitable for melting many batches simultaneously. Melt is directly cooled in the containers. If the substances which hardly wet the melt are employed for the container materials, the samples might be recovered after cooling to room temperature. Method $\mathrm{C}$ is very common technique among glass researchers and gives us most reliable data about glass formation among the methods, but it is a little hard to set up parallel arrangements for high throughput production. Method B in which small scale $(<1 \mathrm{~g})$ batches are melted in small containers had better to be applied to determine glass forming region. In the practical combinatorial research process, the glass forming region should be firstly examined by the method $\mathrm{B}$ and then, the sample glasses should be prepared by the method $\mathrm{C}$ for the evaluation of properties.

Glass forming regions can be determined by the Glass Formation Tester ${ }^{5)}$ (Fig. 3), in which 24 glass batches are melted and cooled in carbon crucibles at one time. One test takes about $1 \mathrm{~h}$ and the possible number of the tests for one working day is $\sim 200$, covering the determination of $2-3$ glass forming regions in a ternary system or of one glass forming region in a 4-component system. The preparation of the glass samples can be carried out using Combinatorial Glass Batch Melting Apparatus.6) The production of the apparatus is $\sim 100$ samples within a working day. The apparatus is equipped with the computer aided CCD video camera system for the identification of the state (the fraction of the transparent part) of the samples and can be used to determine the glass forming regions.

The basic properties of glasses like glass transition temperature, softening temperature, crystallization temperature and liquidus temperature, are measured using a DTA apparatus (MAC Science Co., SLTD, TG-DTA2000SR) equipped with an automatic sample changer. 100 samples can be preset for the maximum and can deal about 15-20 samples a day. It is not a parallel process but continuous operation can make measurement speed up very much.

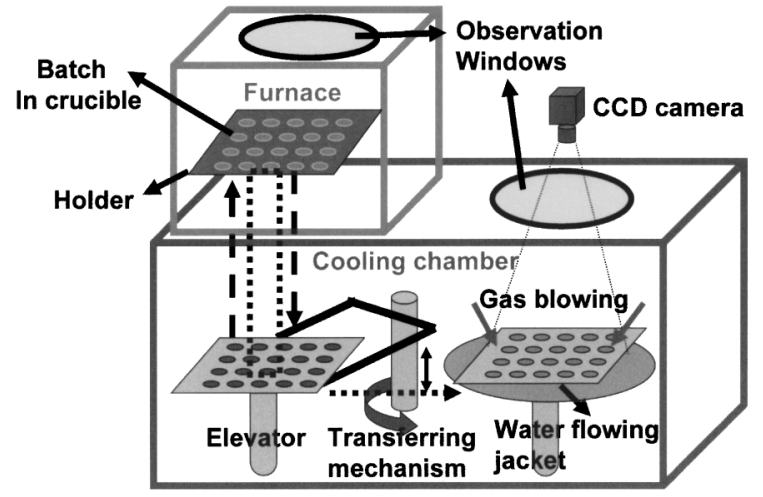

Fig. 3. Combinatorial glass formation tester.

\subsection{Working of the system}

\subsubsection{Preparation of glass batches}

Glass batches are prepared from reagent chemicals using a powder dosing system, Combinatorial Glass Batch Preparation Apparatus consisting of containers, powder feeding mechanisms, an electronic balance and a computer system. The apparatus are distributing chemical powders into crucibles as the guide of the recipe filed in the computer system. The apparatus is not equipped with a mixing mechanism. The mixtures are mixed well in the crucibles using a combinatorial manual mixer. The crucibles containing the mixture are hold between the two rubber lined aluminum plates and rotated for several times.

2.2.2 Determination of glass forming region

Glass forming regions are determined using Combinatorial Glass Formation Tester (Fig. 3) or Combinatorial Glass Batch Melting Apparatus. In Glass Formation Tester, melts are cooled in the crucibles on a water flowing jacket together with $\mathrm{N}_{2}$ gas blowing from above to make the cooling rates comparable as the case of Combinatorial Glass Batch Melting Apparatus, in which melts are poured out and cooled on a metal plate. As the cooling rates of melts in Glass Formation Tester are equivalent to that of Combinatorial Glass Batch Melting Apparatus, the data obtained with these two apparatuses are comparable. The glass formation of cooled melts can be investigated using a CCD camera system. CCD video figures of the samples on a metal plate are stored on a computer and the transparency of the samples is judged by identifying colors of samples in the figures. The results of the identification are filed to compile glass forming region mapping.

\subsubsection{Preparation of samples}

Glass samples are prepared by melting batches using Combinatorial Glass Batch Melting Apparatus. The acceptable quantity of the samples is about $20 \mathrm{~g}$ each. The apparatus is not equipped with a mechanism to homogenize the melts because of the difficulty of installation. When the homogenization of melts is necessary to prepare glasses for the evaluation of optical properties, the samples melted once are crushed to glass frits and melted again by Combinatorial Glass Batch Melting Apparatus. The remelting of the glass frits enhances homogenization of the glasses effectively. A small amount of a sample of a few grams can be prepared using Glass Formation Tester. The video snap shots of casting of melts in crucibles are shown in Fig. 4. The procedures are going as step-(1) for hoisting ten crucibles on the bottom of the furnace, step-(2) for carrying crucibles and making crucibles upside down and step-(3) for casting melts onto a metal plate. 

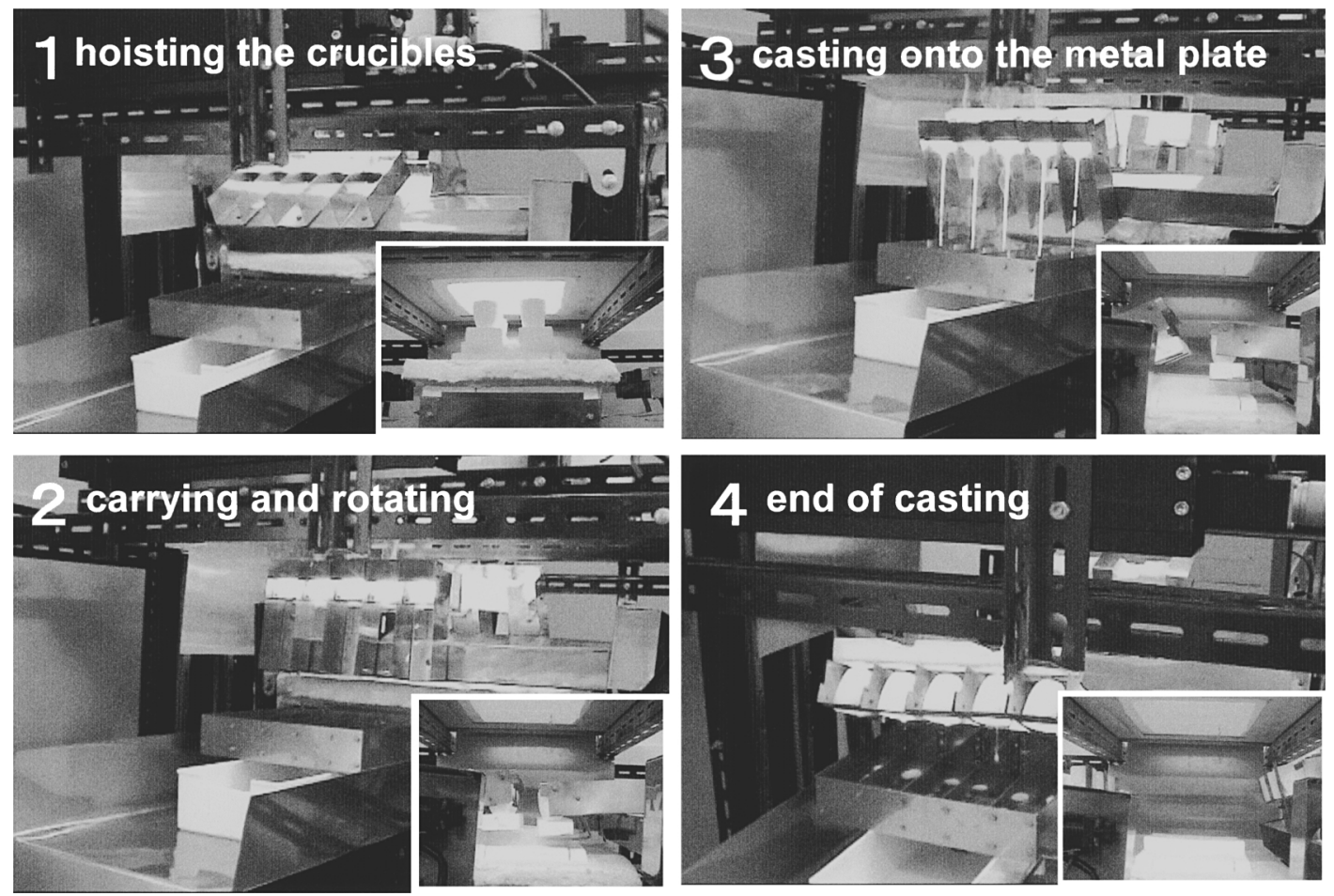

Fig. 4. Casting of glass melts from crucibles on combinatorial glass batch melting apparatus.

\subsection{Advancement of combinatorial glass synthesis system}

The preparation processes of glass samples are working well and a researcher can get about 300 samples within a day working time. The number of samples, 300, is large enough to survey $2-3$ ternary glass forming systems. The current synthesis system can assist the preparation of glass samples by researchers who do not have any background of glass synthesis. To make the system step up, the system should have characterization/evaluation processes to speed up the screening or the optimization of candidate materials.

It is impossible to accelerate DTA measurements by heating samples at a rate faster than $10^{\circ} \mathrm{C} / \mathrm{min}$, that is the common heating rate in glass science and technology. To speed up DTA measurements, a Combinatorial DTA system, in which measurements are made on many samples parallel, should be developed.

The quick evaluation of thermal stability of glasses has been attained by Todoroki et al. using an advanced temperature gradient furnace apparatus, Combinatorial Glass Sample Melter and Annealer. ${ }^{7)}$ Combinatorial Glass Sample Melter and Annealer consists of a temperature gradient tubular furnace, mechanisms to sack up glass melt from a crucible into a glass capillary and a precise positioning system for a capillary including glass sample in the furnace. Heat treatments of a sample glass in a capillary are carried out at various temperatures in temperature gradient atmosphere which is controlled to have a linear relationship with the position along the axis of the tubular furnace. Conventional temperature gradient technique was applied to discrete glass samples, ${ }^{8}$ but Combinatorial Glass Sample Melter and Annealer manages a glass rod sample in a capillary. Time-Temperature-Transformation curves $^{9)}$ can be determined within a week by tracing the temperatures corresponding to the ends of the opaque part of the capillaries in the order of heat treatment times.
3. Advanced preparation method for nanostructures on glass surface

3.1 Development of the method

The anodic oxidation of aluminum has been used as the method to convert the aluminum surface into alumina for the improvement of the chemical durability and is known as the self organization process to generate the porous alumina, where the nanopores are arranged as perpendicular to the substrate. Recently nanostructured materials are attracting great interest for their potentiality leading to the unique functions in the optical properties, electronic properties, magnetic properties and mechanical properties. In these years, the porous anodic alumina has been studied to fabricate the nanostructured materials. ${ }^{10)-14)}$ The anodic oxidation of a thin aluminum film has been studied recently on the sputtered aluminum film on a $\mathrm{Ta}^{15)}$ and an $\mathrm{Si}^{16}$ ) substrate, resulting in the oxidation of the aluminum layer followed by the oxidation of the substrate.

Chu et al. have been studying the formation of the nanostructures on glass surfaces and have developed the new process in which the sputtered alumina film on the conductive layer (tin-doped indium oxide (ITO)) on the glass substrate was converted into the nanostructures by anodic oxidation. ${ }^{17)}$ Chu et al. have also been studying the processes to introduce other compounds into nanopores of the anodic alumina using sol-gel technique and electro deposition. The $\mathrm{TiO}_{2}$ component could be introduced by sol-gel process ${ }^{18), 19)}$ giving the high efficiency photo catalytic function. ${ }^{20)}$ The density of the nanopores of the alumina nanostructures on the glass surface was estimated to be about $1000 \mathrm{Gbit} /$ inch $^{2}$ and the introduction of the magnetic components possessing easy magnetization axis perpendicular to the substrate would be applicable to ultra high density magnetic recording media. The introduction of $\mathrm{Ni}$ metal could be attained by electro deposition process and the magnetization characteristics of the $\mathrm{Ni}$ nanorods were investigated. ${ }^{21)}$ 


\subsection{Details of the method}

\subsubsection{Substrate}

Pure aluminum (99.99\%) was deposited on a soda-limesilica glass substrate coated with ITO and $\mathrm{SiO}_{2}$ protection thin film by RF sputtering technique. The size of the substrate was $20 \times 100 \times 1.1 \mathrm{~mm}$. The thickness of the ITO film and $\mathrm{SiO}_{2}$ film were $100-130 \mathrm{~nm}$ and $\sim 15 \mathrm{~nm}$, respectively. The sheet resistivity of the ITO film was $\sim 20$ ohm. The aluminum layer was deposited at a rate of $\sim 1.5$ $\mathrm{nm} / \mathrm{s}$ in 2.5 cycles $(0.8 \mu \mathrm{m}$ per cycle $)$ under the pressure of $1.0 \times 10^{-5}$ Torr to reach $\sim 2 \mu \mathrm{m}$ in thickness. The sketch of a substrate is illustrated in Fig. 5. The part of the films on the substrate was enlarged and the scale of the figure does not reflect the real dimensions.

3.2.2 Anodic oxidation ${ }^{17}$

The aluminum thin film was anodized by a regulated dc power supply in a $10 \mathrm{vol} \%$ phosphoric acid solution or in a $3 \%$ oxalic acid solution or in a $10 \%$ sulfuric acid solution. The specimens were washed by ultrasonic-cleaning in ethanol for $10 \mathrm{~min}$ and were anodized in phosphoric acid at a voltage of $130 \mathrm{~V}$ at $7^{\circ} \mathrm{C}$, in oxalic acid at $40 \mathrm{~V}$ at $20^{\circ} \mathrm{C}$ and in sulfuric acid at $15 \mathrm{~V}$ at $10^{\circ} \mathrm{C}$. The anodic oxide films prepared in phosphoric, oxalic and sulfuric acid solutions are quoted as phosphoric films, oxalic films and sulfuric films, respectively. The setup for the anodization was shown in Fig. 6 together with a model figure of anodic alumina. Anodic alumina consists of hexagonal cells possessing pores around the centers. Barrier layer is formed at the bottoms of the pores. The anodized specimens were etched in a $5 \mathrm{vol} \%$ phosphoric acid at $30^{\circ} \mathrm{C}$ for $2-15$ min to enlarge the pore size and remove the anodic alumina barrier layers at the bottoms of the pores (to expose the ITO layer to the electrolyte) for the smooth electro deposition of Ni metal.

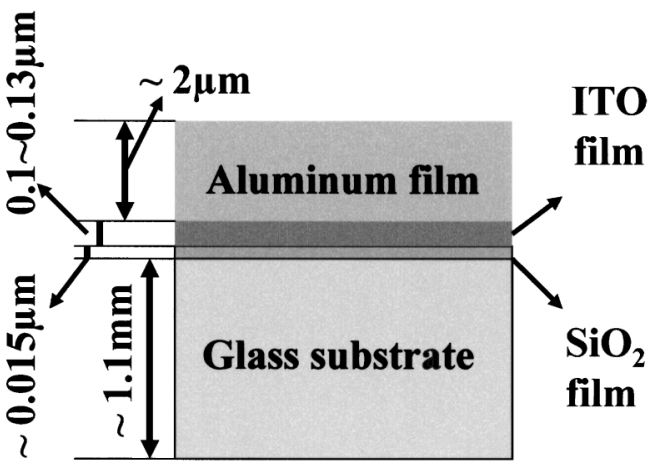

Fig. 5. Illustration of starting specimen.

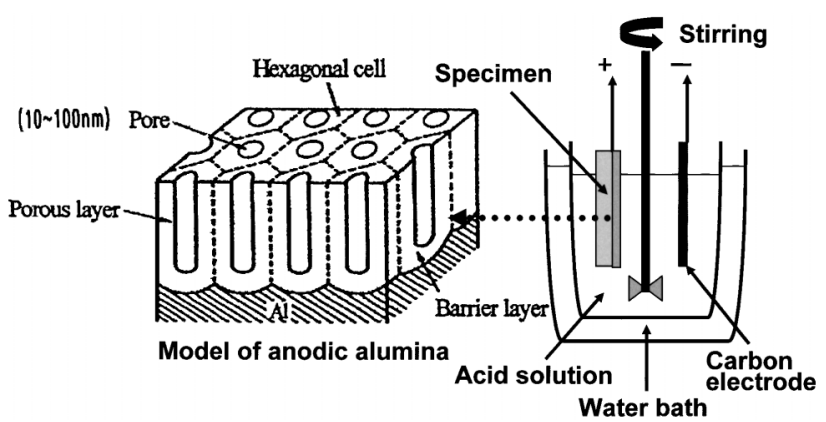

Fig. 6. Model of anodic alumina and set up for anodization.

\subsubsection{How to introduce compounds into pores}

3.2.3.1 Sol-gel process ${ }^{18), 19)}$

The $\mathrm{TiO}_{2}$ sol $(\sim 5 \mathrm{wt} \%)$ was prepared from reagent grade titanium isopropoxide, acetylacetone, ethanol and distilled water at a mole ratio of $1: 1: 20: 3$. The reaction was carried out at room temperature in open air. The mixture was stirred for $2 \mathrm{~h}$ and stored in a brown bottle at room temperature. Prior to the experiments, the sol solution was aged for 1 week-1 month. Though the color of the sol changed gradually from light yellow to dark yellow, nothing precipitated and the solution was very stable.

In the introduction of $\mathrm{TiO}_{2}$ sol into the nanopores of the anodic alumina, the specimens were first immersed in ethanol for $10 \mathrm{~min}$ and then dipped in the $\mathrm{TiO}_{2}$ sol at room temperature for $20 \mathrm{~min}$. After $20 \mathrm{~min}$ dipping, the specimens were lifted up slowly from the solution. The $\mathrm{TiO}_{2}$ sol on the back side of the specimen was washed away with ethanol. The specimens dipped in the $\mathrm{TiO}_{2}$ sol were dried in the air at room temperature for more than $2 \mathrm{~h}$. To obtain porous alumina/titania structure, the specimens were heated in the furnace at $100^{\circ} \mathrm{C}$ for $1 \mathrm{~h}$ and at $400^{\circ} \mathrm{C}$ for $2 \mathrm{~h}$ where the heating rate was $2^{\circ} \mathrm{C} / \mathrm{min}$. To get $\mathrm{TiO}_{2}$ nanotube array, the anodic alumina skeletons were removed with the etching by $5 \%$ phosphoric acid and $2 \% \mathrm{CrO}_{3}$ solution at $75^{\circ} \mathrm{C}$ for $5-8 \mathrm{~min}$.

\subsubsection{Electro deposition process ${ }^{21)}$}

The electrolyte solution for the $\mathrm{Ni}$ electro deposition consisted of $0.38 \mathrm{M} \mathrm{NiSO}_{4}, 0.13 \mathrm{M} \mathrm{NiCl}_{2}, \quad 0.65 \mathrm{M} \mathrm{H}_{3} \mathrm{BO}_{4}$ and $60 \mathrm{ppm} \mathrm{CH}_{3}\left(\mathrm{CH}_{2}\right)_{11} \mathrm{OSO}_{3} \mathrm{Na}$, and was adjusted to $\mathrm{pH}$ of 5.2 with a $20 \% \mathrm{NaOH}$ solution. The specimens were immersed in distilled water for $1 \mathrm{~min}$ to push out the air in the pores with applying ultrasonic wave prior to the electro deposition. The $\mathrm{Ni}$ electro deposition was performed at $1.0-1.5 \mathrm{~V}$ and $30^{\circ} \mathrm{C}$ for $2-10 \mathrm{~min}$, with stirring. The counter electrode was a pure nickel plate. To get $\mathrm{Ni}$ nanorods, the alumina skeletons of the specimens were removed with the etching by $5 \% \mathrm{NaOH}$ solution

3.3 Nanostructures on the glass surface ${ }^{17)}$

The FESEM photos (FESEM; S-500, Hitachi with an energy-dispersion X-ray analyzer, EDXA) of the anodic alumina prepared under various conditions were shown in Fig. 7. The details of the preparation processes were given in the reference. ${ }^{21}$ The pore sizes of the phosphoric film were $120-180 \mathrm{~nm}$ in diameter after 30 min pore widening treatment. The pore diameters of the anodic film were $40-50 \mathrm{~nm}$ in the oxalic film and 5-8 $\mathrm{nm}$ for the sulfuric film. The pores were arranged as perpendicular to the glass substrate. The specimen became transparent after the anodization. The transmission spectrum of the phosphoric film was measured by a spectrophotometer, U-3500 (Hitachi model) and is given in Fig. 8. The anodic alumina film on the glass gave $\sim 90 \%$ transmission in visible region. The waving of the spectra originated from the interference taking place within the specimen.

3.4 Introduction of $\mathrm{TiO}_{2}$ sol into nanopores ${ }^{18)}$

Figure 9 shows the schematic illustrations, the photos of the specimens and the SEM photos in the process for the synthesis of the $\mathrm{TiO}_{2}$ nanotube array on a glass. After widening of the pores, the sol was introduced into pores. The SEM photo indicated that the inside wall of the pores were coated with the sol. The photo showed that the transparency was kept after the impregnation. The transmission spectrum was given in Fig. 8 as " $\mathrm{Al}_{2} \mathrm{O}_{3} / \mathrm{TiO}_{2}$ Nanostructure," showing the transmission of $30-80 \%$ in visible region. The specimen was converted into the $\mathrm{TiO}_{2}$ nanotube array after dissolution of the alumina skeletons. The dimensions of the nanutubes were $\sim 200 \mathrm{~nm}$ in outside diameter and $\sim 140 \mathrm{~nm}$ in inside diameter. The length of the nanotubes correspond to the thickness of the anodic alumina 


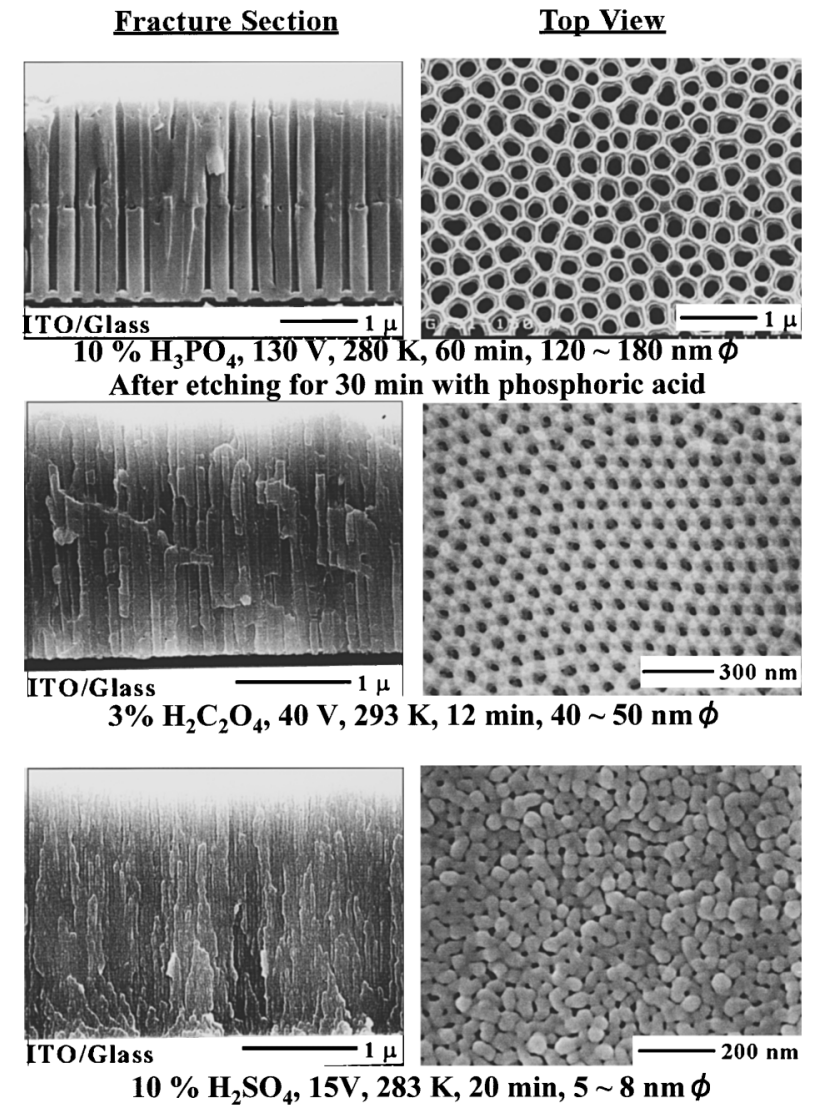

Fig. 7. SEM photos of the nanostructures of anodic alumina on glass surfaces.

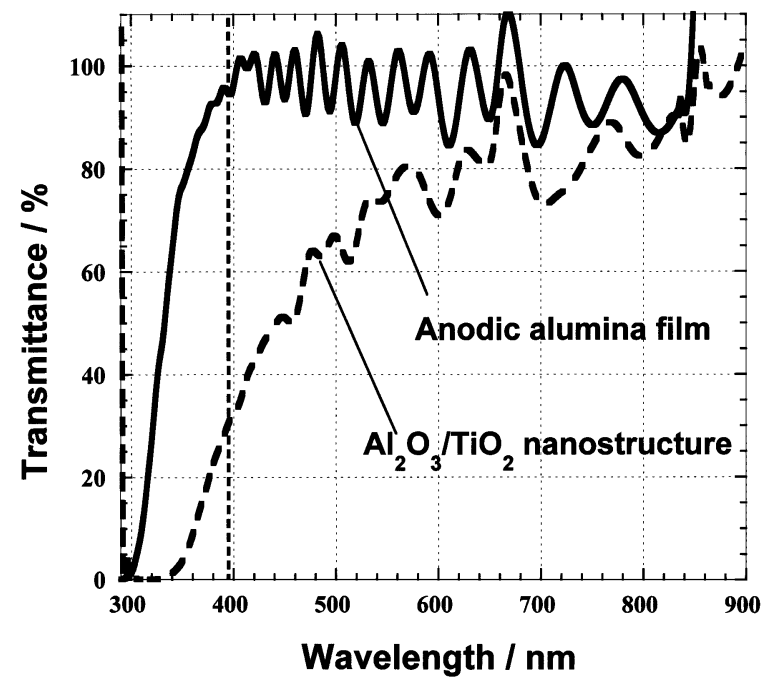

Fig. 8. Transmission curves of the specimens.

film and were $\sim 3 \mu \mathrm{m}$. The translucency of the specimen was kept but the transmittance decreased a little as shown in Fig. 8, where the spectrum was quoted as " $\mathrm{TiO}_{2}$ Nanotube Array." The transmittance was $\sim 60 \%$ in visible region and decreased gradually in UV region. The surface area of the specimen was estimated to increase by $\sim 45$ times from the calculation based on the simple geometry. The $\mathrm{TiO}_{2}$ formed in the process was identified to be anatase

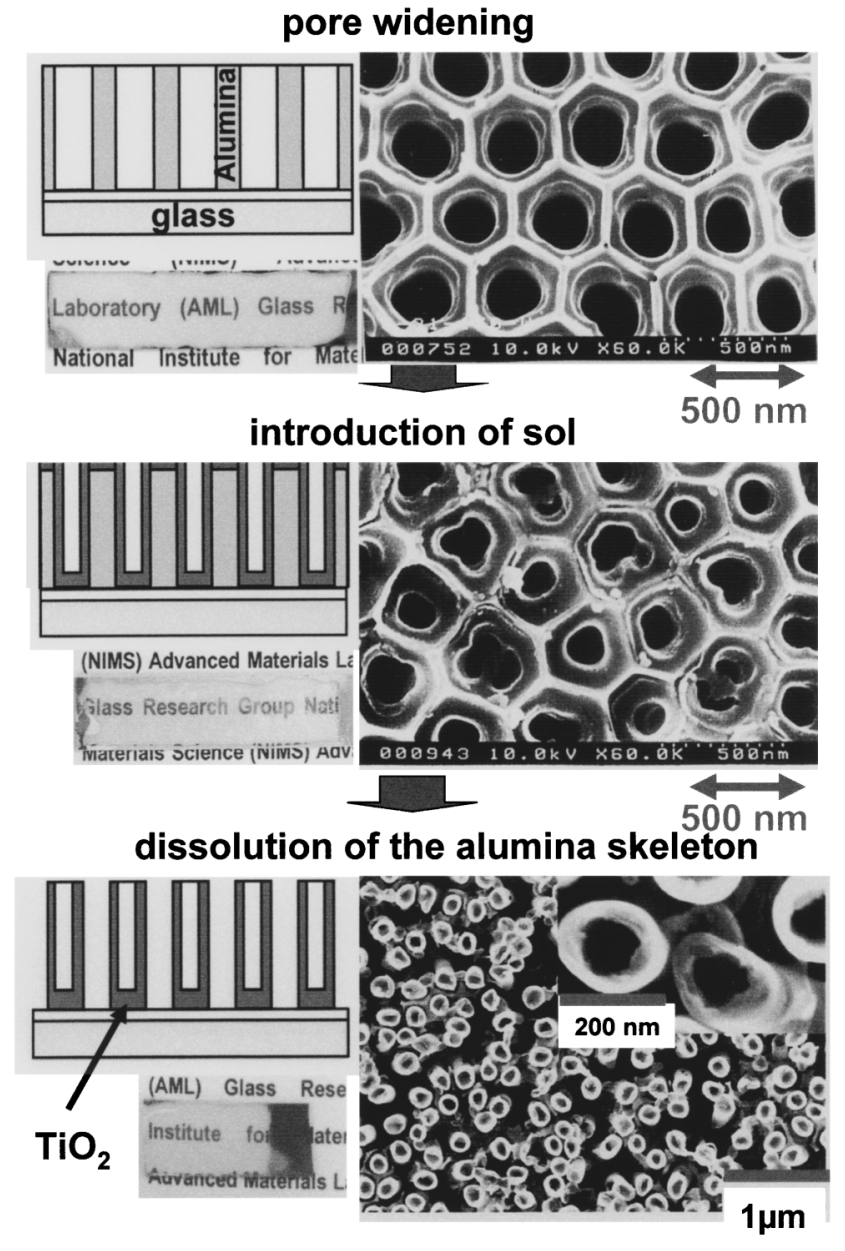

Fig. 9. SEM photos, photos and model illustrations of the specimens before/after the $\mathrm{TiO}_{2}$ sol impregnation.

by using an X-ray diffractometer (XRD; RINT-2000/PC) operated on $\mathrm{Cu} \mathrm{K} \alpha$ at a generation condition of $40 \mathrm{~V} / 40 \mathrm{~mA}$.

The evaluation of the photo catalysis of the $\mathrm{TiO}_{2}$ nanotube array was performed by monitoring the volume of the $\mathrm{CO}_{2}$ gas released in the decomposition of acetaldehyde. ${ }^{20)}$ The popular and high performance photo catalysis, Degussa P25, was used as the reference sample. The UV light source was a $200 \mathrm{~W} \mathrm{Hg}$-Xe lamp possessing the peak emission at $365 \mathrm{~nm}$. The relative value of the volume of the released $\mathrm{CO}_{2}$ gas was plotted against the irradiation time of UV light in Fig. 10. All the data were normalized to the specimen area of $1 \mathrm{~cm}^{2} .100 \%$ level corresponds to the decomposition of all of the acetaldehyde input. The impregnated anodic film on the glass gave photo catalytic function better than Degussa P-25. The impregnated phosphoric film showed the decomposition rate larger by $\sim 13$ times than that of Degussa P-25. Oxalic film case gave the intermediate decomposition rate. The catalytic function of a self supporting $\mathrm{TiO}_{2}$ nanotube array has not yet been finished, but would be better for the increase of the surface area. The sulfuric film impregnated with $\mathrm{TiO}_{2}$ sol did not seem to have open pores and was not taken in the catalytic function test. The reason why the oxalic film case showed decomposition rate lower than that of the phosphoric case could be that oxalic film small pores restricted light irradiation or gas migration within pores.

3.5 Introduction of $\mathrm{Ni}$ into nanopores ${ }^{21}$ )

In Fig. 11, the cross sectional SEM photos were 


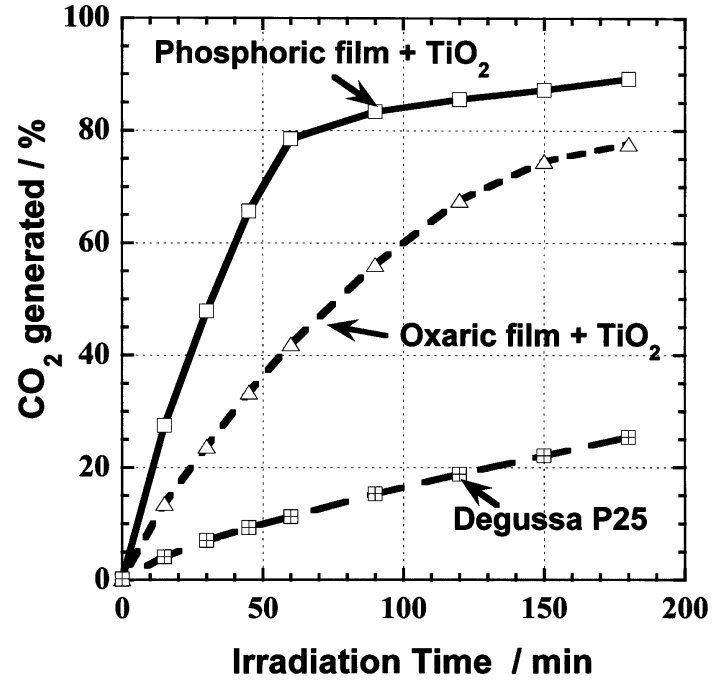

Fig. 10. Relation between $\mathrm{CO}_{2}$ gas generation and $\mathrm{UV}$ irradiation time.

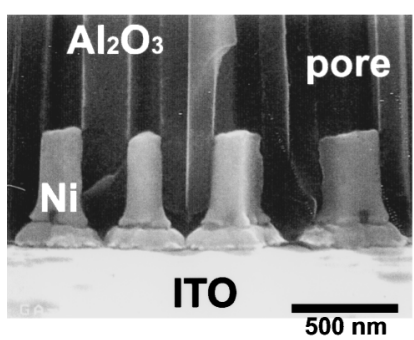

phosphoric film

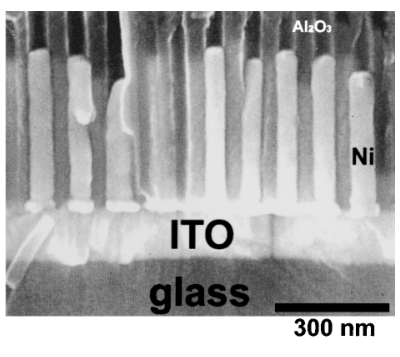

oxalic film
Fig. 11. Cross sectional SEM photos of the specimens introduced with $\mathrm{Ni}$ by electro deposition.

shown for the cases of the phosphoric film and oxalic film. $\mathrm{Ni}$ deposition was performed directly on an ITO film. When the anodic alumina barrier layer remained, the electro deposition could not be performed. The SEM photos of the Ni nanorod arrays, which were obtained after dissolution of anodic alumina skeletons, were summarized in Fig. 12. The phosphoric film, the oxalic film and the sulfuric film gave the nanorods of $\sim 150 \mathrm{~nm}, \sim 50 \mathrm{~nm}$ and $\sim 18 \mathrm{~nm}$ in diameter, respectively. Magnetization curves of the $\mathrm{Ni}$ nanorods were measured by a vibrating sample magnetometer (USM; Lake Shore model 7300). The Fig. 13 shows the magnetization curves for the $\mathrm{Ni}$ nanorod embedded in the anodic alumina skeletons. In the figures, A. R. indicates the average aspect ratio of the $\mathrm{Ni}$ nanorods. The top one is for the phosphoric film, the middle one for the oxalic film and the bottom one for the sulfuric film. The figure indicated that the easy axis of magnetization changed from in-plane to perpendicular direction with increase of the aspect ratio of the nanorod. In other words, the magnetization perpendicular to the substrate became dominant over the in-plane magnetization. Taking that the density of the pores of the anodic films was about 1000 Gbit/inch ${ }^{2}$ into account, the anodic films embedded with appropriate magnetic substances could be applied to the ultra high density magnetic recording media.

3.6 Potentiality of anodic oxidation of sputtered aluminum

The anodic oxidation of a sputtered aluminum on a glass

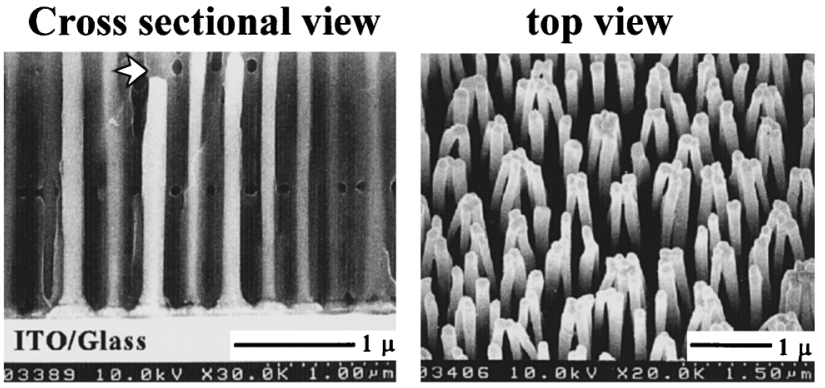

$10 \% \mathrm{H}_{3} \mathrm{PO}_{4}: \sim \phi 150 \mathrm{~nm} \times 2.1 \mu \mathrm{m}$, Aspect Ratio -- 14 (22)
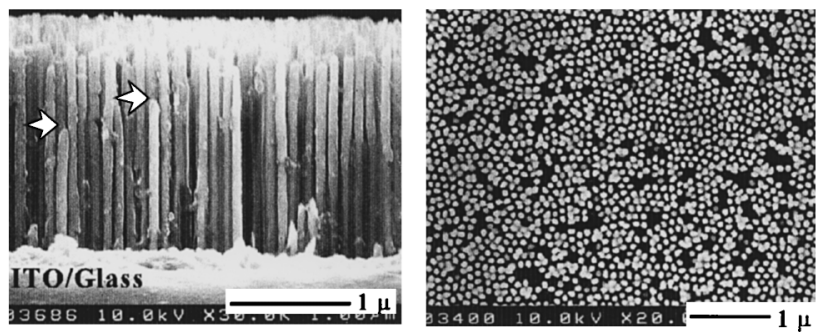

$3 \% \mathrm{H}_{2} \mathrm{C}_{2} \mathrm{O}_{4}: \sim \$ 50 \mathrm{~nm} \times 1.8 \mu \mathrm{m}$, Aspect Ratio -- 36 (66)

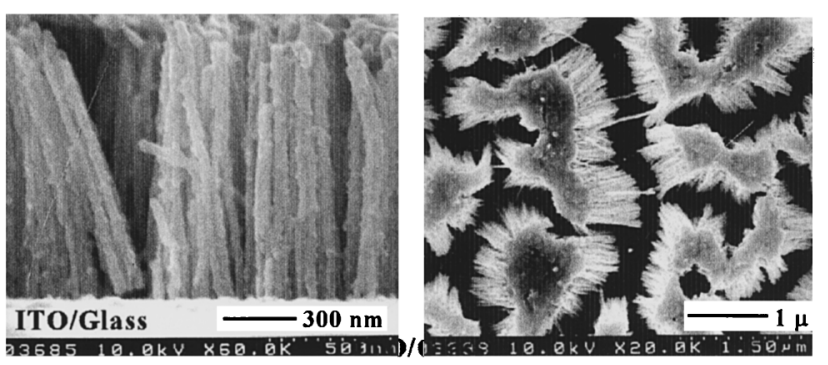

$10 \% \mathrm{H}_{2} \mathrm{SO}_{4}: \sim \phi 18 \mathrm{~nm} \times 1.2 \mu \mathrm{m}$, Aspect Ratio -- 67 (183)

Fig. 12. SEM photos of Ni nanorod arrays prepared using various anodic alumina films.

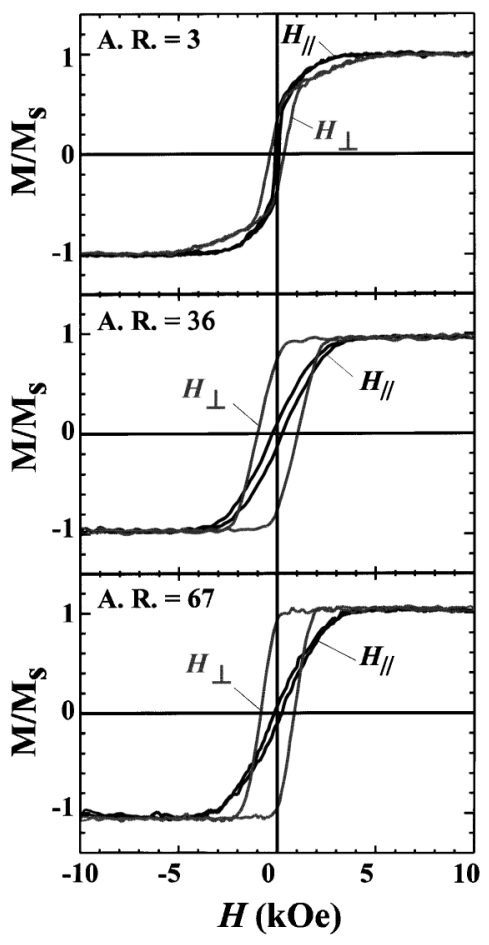

Fig. 13. Magnetization characteristics of the Ni nanorod arrays. 
substrate was fruitful process to build up nanostructures on a glass surface. The introduction of compounds into pores could be attained with sol-gel processing and electro deposition technique. The embedding of components into nanopores could endow the glasses with photo catalytic function and would be applicable to ultra high density magnetic recording. The photo catalyst prepared in this study showed the decomposition rate of acetaldehyde larger by one order of magnitude than that of the Degussa P-25. The anodic oxidation process, the sol-gel coating process and the electro deposition process are principally applicable to general curved substrates and large substrates. The new process does not require the special and expensive apparatuses like high power lasers, nanometer range precise manipulators etc and is a simple mass-production type technique. Therefore the process is believed to have an advantage over the other nanomachining processes in the applications and the commercial productions.

\section{Conclusion}

The combinatorial glass synthesis system was effective to speed up preparation of glass samples, leading to shortening of the period of developments of bulk functional glasses. The reproducibility of the sample synthesis conditions in the combinatorial system was effective to produce reliable data of glass formation, resulting in the filing of reliable characterization data of glass samples. The combinatorial system would assist researchers in the fields other than glass science/technology to prepare preparation of glass samples and would promote the developments of various functional glasses.

The anodic oxidation of sputtered aluminum on glass surfaces was useful method to prepare nanostructures on glass surfaces. The introduction of compounds into nanopores could be performed using sol-gel technique or electro deposition technique. The coating of $\mathrm{TiO}_{2}$ on the walls of nanopores would produce high performance photo catalytic function and the introduction of paramagnetic metal or alloys might give high density magnetic recording media. The anodic alumina embedded with $\mathrm{TiO}_{2}$ showed the very quick photo catalytic decomposition rate of acetaldehyde lager by about 13 times than that of high performance commercial $\mathrm{TiO}_{2}$ photo catalysis, $\mathrm{P}-25$. Moreover, this technique is believed to have potentials on applications to the preparation of various functional glasses like solar cells, photonic crystals etc.

\section{References}

1) For example, Yamane, M., Inoue, S., Suga, Y. and Kawazoe, H., J. Non-Cryst. Solids, Vol. 56, pp. 87-92 (1983).

2) For example, Inoue, S., Shimizugawa, Y., Nukui, A. and Maeseto, T., J. Non-Cryst. Solids, Vol. 189, pp. 36-42 (1995).

3) Inoue, S., Todoroki, S., Matsumoto, T., Hondo, T., Araki, T. and Watanabe, Y., Appl. Surf. Sci., Vol. 189, pp. 327-332 (2002).

4) For example, Van Dover, R. B., Schneemeyer, L. F. and Fleming, R. M., Nature, Vol. 392, pp. 162-164 (1998).

5) Inoue, S., Todoroki, S., Matsumoto, T., Hondo, T., Araki, T. and Tsuchiya, T., MRS Symp. Proc., Vol. 700, pp. 201-208 (2002).

6) Matsumoto, T., Inoue, S., Todoroki, S., Hondo, T., Araki, T. and Watanabe, Y., Appl. Surf. Sci., Vol. 189, pp. 234-240 (2002).

7) Todoroki, S., Inoue, S. and Matsumoto, T., Appl. Surf. Sci., Vol. 189, pp. 241-244 (2002); Todoroki, S., Matsumoto, T. and Inoue, S., MRS Symp. Proc., Vol. 700, pp. 209-214 (2002).

8) Grauer, O. H. and Hamilton, E. H., J. Res. National Bureau of Standards, Vol. 44, pp. 495-502 (1950).

9) For example, Uhlmann, D. R., J. Non-Cryst. Solids, Vol. 7, pp. 337-348 (1972).

10) Huczko, A., Appl. Phys., Vol. 70, pp. 365-376 (2000).

11) Kyotani, T., Pradhan, B. K. and Tomita, A., Bull. Chem. Soc. Jpn., Vol. 72, pp. 1957-1970 (1999).

12) Zhang, M., Bando, Y., Wada, K. and Kurashima, K., J. Mater. Sci. Lett., Vol. 18, pp. 1911-1913 (1999).

13) Lakshmi, B. B., Dorhout, P. K. and Martin, C. R., Chem. Mater., Vol. 9, pp. 857-862 (1997).

14) Masuda, H. and Fukuda, K., Science, Vol. 268, pp. 1466-1468 (1995).

15) For example, Mozalev, A., Surganov, A. and Imai, H., Electrochim. Acta, Vol. 46, pp. 2825-2834 (2001).

16) For example, Crouse, D., Lo, Yu-Huwa, Miller, A. E. and Crouse, M., Appl. Phys. Lett., Vol. 76, pp. 49-51 (2000).

17) Chu, S. Z., Wada, K., Inoue, S. and Todoroki, S., J. Electrochem. Soc., Vol. 149, pp. B321-B327 (2002).

18) Chu, S. Z., Wada, K., Inoue, S. and Todoroki, S., Chem Mater., Vol. 14, pp. 266-272 (2002).

19) Chu, S. Z., Wada, K. and Inoue, S., Adv. Mater., Vol. 14, pp. 1752-1756 (2002).

20) Chu, S. Z., Inoue, S., Wada, K., Li, D. and Haneda, H., J. Mater. Chem., Vol. 13, pp. 866-870 (2003).

21) Chu, S. Z., Wada, K., Inoue, S. and Todoroki, S., Chem. Mater., Vol. 14, pp. 4595-4602 (2002).

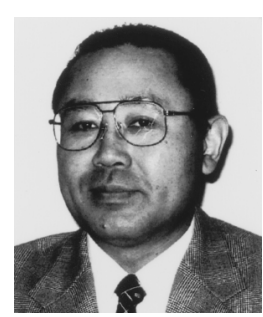

Satoru Inoue received his Bachelor degree in engineering from Tokyo Institute of Technology (TIT) in 1975. He advanced to Master course of TIT and got his Master degree in chemical engineering in 1977. He earned his $\mathrm{Ph}$. D. degree in chemical engineering from TIT in 1985 while he worked as a research associate at Faculty of Engineering of TIT from 1977 to 1989 . He joined National Institute for Research in Inorganic Materials as a senior researcher of 9 th research group in 1989. He is now the director of Functional Glass Group at Advanced Materials Laboratory of National Institute for Materials Science. 